Journal of Universal Mathematics

Vol.4 No.1 PP.34-41 (2021)

ISSN-2618-5660

DOI: $10.33773 /$ jum. 823084

\title{
$\lambda$-STATISTICAL SUPREMUM-INFIMUM AND $\lambda$-STATISTICAL CONVERGENCE
}

\author{
M. ALTINOK, U. KAYA AND M. KUCUKASLAN
}

\begin{abstract}
In this paper, we are going to define $\lambda$-statistical supremum and $\lambda$-statistical infimum for real valued sequence $\tilde{x}=\left(x_{n}\right)_{n \in \mathbb{N}}$ by considering $\lambda$-statistical upper and lower bounds, respectively. After giving some basic properties of these new notations, then we will give a necessary and sufficient condition for to existance of $\lambda$-statistical convergence of the real valued sequence.
\end{abstract}

\section{Introduction and Main RESUlts}

The notion of statistical convergence is defined by Fast and Steinhaus at the same time in [4] and [9], independently. In [10], Zygmund gave a name almost convergence to this concept and established a relation between statistical convergence and strong summability of sequences. Especially in [8], Schoenberg gave matrix characterization of statistical convergence.

Let us consider strictly increasing sequence of natural numbers $\lambda=\left(\lambda_{n}\right)_{n \in \mathbb{N}}$ such that

$$
\lim _{n \rightarrow \infty} \lambda_{n}=\infty .
$$

The collection of all this kind of sequences will be denoted by $\Lambda$.

For any sequence $\lambda=\left(\lambda_{n}\right)$ from $\Lambda, \lambda$-density of a subset $K$ of natural numbers $\mathbb{N}$ can be defined as follows, if the following limit exists

$$
\delta_{\lambda}(K):=\lim _{n \rightarrow \infty} \frac{\left|K_{n}^{\lambda}\right|}{\lambda_{n}}
$$

where $K_{n}^{\lambda}:=\left\{k \leq \lambda_{n}: k \in K\right\}$ and the symbol $|$.$| denotes the cardinality of inside$ set.

A real number sequence $\tilde{x}=\left(x_{n}\right)_{n=1}^{\infty}$ is called $\lambda$-statistical convergent to $x_{0}$, if provided that for every $\varepsilon>0$ the set

$$
K(\varepsilon)=\left\{n \in \mathbb{N}:\left|x_{n}-x_{0}\right| \geq \varepsilon\right\}
$$

has zero $\lambda$-density. In this case, we write $s t_{\lambda}-\lim x=L$.

Date: November, 2020.

2000 Mathematics Subject Classification. 40A35, 11B05, 11425.

Key words and phrases. Statistical supremum-infimum, Statistical convergence, Natural density. 
The case $\lambda_{n}=n$ in (1.1) is coincide with natural density and $\lambda$-statistical convergence is coincide with statistical convergence.

Over the years, some new concepts are defined with the help of natural density. One of them is statistical supremum and statistical infimum. In [6], Küçükaslan and Altmok defined statistical supremum(st-sup) and statistical infimum(st-inf) for real valued sequences. Also, it is given that equality of st-sup and st-inf for a sequence $\tilde{x}=\left(x_{n}\right)_{n \in \mathbb{N}}$ is necessary and sufficient for the existance of statistical convergence of a sequence [6]. After this work, generalizations of this concept is investigated for $A$-statistical convergence in [1], ideal convergence in [2] and porosity convergence in [3].

In this paper some results given in [6], [1] and [2] will be generalized by considering $\lambda$-density. Also, necessary and sufficient condition will be given for to existance of $\lambda$-statistical convergence of a real valued sequence.

Definition 1. ( $\lambda$-statistical lower and upper bound) Let $\tilde{x}=\left(x_{n}\right)$ be a sequence of real numbers.

(i) $l \in \mathbb{R}$ is an $\lambda$-statistical lower bound of $\tilde{x}=\left(x_{n}\right)$ if the set $\left\{n: x_{n}<l\right\}$ has zero $\lambda$-density.

(ii) $m \in \mathbb{R}$ is an $\lambda$-statistical upper bound of $\tilde{x}=\left(x_{n}\right)$ if the set $\left\{n: x_{n}>m\right\}$ has zero $\lambda$-density.

The set of $\lambda$-statistical lower and upper bounds of a sequence $x=\left(x_{n}\right)$ will be denoted by $L_{s t_{\lambda}}(\tilde{x})$ and $U_{s t_{\lambda}}(\tilde{x})$, respectively. In usual case, we are going to use $L(\tilde{x})$ and $U(\tilde{x})$ for the sets of usual lower and upper bounds, respectively.

Remark 1.1. (i) If $m$ is usual upper bound, then it is $\lambda$-statistical upper bound.

(ii) If $l$ is usual lower bound, then it is $\lambda$-statistical lower bound.

Let us consider the sequence which states that the inverse of the Remark 1.1 is not true. To see this let us consider a set $A \subset \mathbb{N}$ such that $\delta_{\lambda}(A)=0$. Hence if we take a sequence $\tilde{x}=\left(x_{n}\right)$ as

$$
x_{n}= \begin{cases}1, & n \notin A, \\ (-1)^{n} n, & n \in A .\end{cases}
$$

then it is clear that $\left(x_{n}\right)$ is not bounded but it has $\lambda$-statistical upper and $\lambda$ statistical lower bound.

Remark 1.2. (i) Assume that $l \in L_{s t_{\lambda}}(\tilde{x})$ and $l^{\prime}<l$. Then, $l^{\prime} \in L_{s t_{\lambda}}(\tilde{x})$.

(ii) Assume that $m \in U_{s t_{\lambda}}(\tilde{x})$ and $m<m^{\prime}$. Then, $m^{\prime} \in L_{s t_{\lambda}}(\tilde{x})$.

Proof. Since $(i)$ and $(i i)$ are very similar in type, then, let's just prove here only (i) Let $l \in L_{s t_{\lambda}}(\tilde{x})$ and $l^{\prime}<l$. Hence, following inclusion

$$
\left\{n: x_{n}<l^{\prime}\right\} \subset\left\{n: x_{n}<l\right\}
$$

holds and this inclusion implies that

$$
\left|\left\{n: x_{n}<l^{\prime}\right\}\right| \leq\left|\left\{n: x_{n}<l\right\}\right|
$$

So, the hereditary property of $\lambda$-statistical density implies that $l^{\prime} \in L_{s t_{\lambda}}(\tilde{x})$.

As a result of this remark, we can say that if a sequence has a $\lambda$-statistical upper bound or $\lambda$-statistical lower bound then it has infinitely many $\lambda$-statistical lower or $\lambda$-statistical upper bound. 
Theorem 1.3. The sets $L_{s t_{\lambda}}(\tilde{x})$ and $U_{s t_{\lambda}}(\tilde{x})$ are closed subset of $\mathbb{R}$ (with standart metric) for any sequence $\tilde{x}=\left(x_{n}\right)$ and $\lambda \in \Lambda$.

Proof. Let $a \in \mathbb{R} \backslash L_{s t_{\lambda}}(\tilde{x})$ be an arbitrary number such that

$$
\delta_{\lambda}\left(\left\{n: x_{n}<a\right\}\right)>0
$$

holds. Now, assume that $a$ is not inner point of the set $\mathbb{R} \backslash L_{s t_{\lambda}}(\tilde{x})$. It means that for all $\varepsilon>0$,

$$
(a-\varepsilon, a+\varepsilon) \cap L_{s t_{\lambda}}(\tilde{x})
$$

contains a common point $l \in \mathbb{R}$. Here, there is two cases: $\left(i^{\prime}\right) a<l$ and $\left(i i^{\prime}\right) l<a$. From Remark $1.2(i)$ we know that $\left(i^{\prime}\right)$ is not true.

Hence, $a-\varepsilon<l<a$ should satisfied for all $\varepsilon>0$. Because of following inclusion

$$
\left\{n: x_{n}<a-\varepsilon\right\} \subset\left\{n: x_{n}<l\right\},
$$

we have

$$
\delta_{\lambda}\left(\left\{n: x_{n}<a-\varepsilon\right\}\right)=0 .
$$

If we take $\varepsilon=\frac{1}{n}$ in (1.2) and consider

$$
\left\{n: x_{n}<a\right\}=\bigcup_{n=1}^{\infty}\left\{n: x_{n}<a-\frac{1}{n}\right\}
$$

then, we have

$$
\delta_{\lambda}\left(\left\{n: x_{n}<a\right\}\right) \leq \sum_{n=1}^{\infty} \delta_{\lambda}\left(\left\{n: x_{n}<a-\frac{1}{n}\right\}\right) .
$$

The right hand side in above inequality is zero because of (1.2). Hence, $\delta_{\lambda}(\{n$ : $\left.\left.x_{n}<a\right\}\right)=0$. This contradicts with $a \notin \mathbb{R} \backslash L_{s t_{\lambda}}(\tilde{x})$. Therefore, there exists a $\varepsilon_{0}>0$ such that

$$
\left(a-\varepsilon_{0}, a+\varepsilon_{0}\right) \subset \mathbb{R} \backslash L_{s t_{\lambda}}(\tilde{x})
$$

holds.

Definition 2. ( $\lambda$-statistical infimum, $\lambda$-statistical supremum) A number $s \in \mathbb{R}$ is called $\lambda$-statistical infimum (or $\lambda$-statistical supremum) of the sequence $\tilde{x}=\left(x_{n}\right)$, if it is supremum (or infimum) of $L_{s t_{\lambda}}(\tilde{x})$ (or $U_{s t_{\lambda}}(\tilde{x})$ ). That is

$$
s t_{\lambda}-\inf \left(x_{n}\right):=\sup L_{s t_{\lambda}}(\tilde{x}), s t_{\lambda}-\sup \left(x_{n}\right):=\inf U_{s t_{\lambda}}(\tilde{x})
$$

Theorem 1.4. For any $\lambda \in \Lambda$ and real valued sequence $\tilde{x}=\left(x_{n}\right)$ following inequalities holds:

$$
\inf x_{n} \leq s t_{\lambda}-\inf x_{n} \leq s t_{\lambda}-\sup x_{n} \leq \sup x_{n}
$$

Proof. It is well known that $\inf x_{n} \leq x_{n} \leq \sup x_{n}$ holds for all $n \in \mathbb{N}$. Hence, $\lambda$-density of the following sets

$$
\left\{n: x_{n}<\inf x_{n}\right\}=\emptyset,\left\{n: \sup x_{n}<x_{n}\right\}=\emptyset
$$

are zero. So, $\inf x_{n} \in L_{s t_{\lambda}}\left(x_{n}\right)$ and $\sup x_{n} \in U_{s t_{\lambda}}\left(x_{n}\right)$ hold. This implies that

$$
\inf x_{n} \leq s t_{\lambda}-\inf x_{n} \text { and } s t_{\lambda}-\sup x_{n} \leq \sup x_{n}
$$


satisfied. For complete the proof let $l \in L_{s t_{\lambda}}(\tilde{x})$ be an arbitrary element. It is clear from Remark 1.2 that $l \leq m$ holds for all $m \in U_{s t_{\lambda}}(\tilde{x})$.

So $l \leq \inf U_{s t_{\lambda}}(\tilde{x})$ and $\sup L_{s t_{\lambda}}(\tilde{x}) \leq \inf U_{s t_{\lambda}}(\tilde{x})$ holds. This implies that

$$
s t_{\lambda}-\inf x_{n} \leq s t_{\lambda}-\sup x_{n}
$$

satisfied for any $\lambda \in \Lambda$.

Theorem 1.5. Let $\tilde{x}=\left(x_{n}\right)$ be a sequence and $\lambda \in \Lambda$ be an arbitrary sequence. Then, (1) $s t_{\lambda}-\sup x_{n}=l$ if and only if

$$
\begin{aligned}
& \text { (i) } \delta_{\lambda}\left(\left\{n: x_{n} \leq l+\varepsilon\right\}\right)=1, \\
& \text { (ii) } \delta_{\lambda}\left(\left\{n: l-\varepsilon<x_{n}\right\}\right) \neq 0 .
\end{aligned}
$$

(2) $s t_{\lambda}-\inf x_{n}=m$ if and only if

$$
\begin{aligned}
& \text { (i) } \delta_{\lambda}\left(\left\{n: m-\varepsilon \leq x_{n}\right\}\right)=1, \\
& \text { (ii) } \delta_{\lambda}\left(\left\{n: m+\varepsilon>x_{n}\right\}\right) \neq 0 .
\end{aligned}
$$

Proof. (1) Assume that $s t_{\lambda}-\sup x_{n}=l$. Then, $l=\inf U_{s t_{\lambda}}(\tilde{x})$. This implies that

$$
\text { a) } l \leq s, \forall s \in U_{s t_{\lambda}}(\tilde{x})
$$

$$
\text { b) } \forall \varepsilon>0, \exists s^{\prime} \in U_{s t_{\lambda}}(\tilde{x}) \ni s^{\prime} \leq l+\varepsilon
$$

holds. From Remark 1.2, $l+\varepsilon \in U_{s_{\lambda}}(\tilde{x})$. So, $(i)$ is satisfied. Assume that there exists an $\varepsilon_{0}>0$ such that $(i i)$ is not true:

$$
\delta_{\lambda}\left(\left\{n: l-\varepsilon_{0}<x_{n}\right\}\right)=0 .
$$

This assumption implies that $l-\varepsilon_{0} \in U_{s t_{\lambda}}(\tilde{x})$. Since $l-\varepsilon_{0}<l$, then this is a contradiction to assumption $l=\inf U_{s t_{\lambda}}(\tilde{x})$.

Now assume that $(i)$ and $(i i)$ are satisfied. This implies that $l+\varepsilon \in U_{s t_{\lambda}}(\tilde{x})$ and $l-\varepsilon \notin U_{s t_{\lambda}}(\tilde{x})$ for all $\varepsilon>0$, respectively. As a result of this facts we have

$$
U_{s t_{\lambda}}(\tilde{x})=[l+\varepsilon, \infty),
$$

for any $\varepsilon$. Hence, $l=s t_{\lambda}-\sup x_{n}$.

The proof of (2) is very similar to (1). So, it ommitted here for this similarity.

Theorem 1.6. If $\lim _{n \rightarrow \infty} x_{n}=x_{0}$, then

$$
s t_{\lambda}-\inf x_{n}=s t_{\lambda}-\sup x_{n}=x_{0} .
$$

Proof. Assume that $\lim _{n \rightarrow \infty} x_{n}=x_{0}$ holds. It means that $\forall \varepsilon>0$, there exists $n_{0} \equiv$ $n_{0}(\varepsilon) \in \mathbb{N}$ such that

$$
\left|x_{n}-x_{0}\right|<\varepsilon
$$

is satisfied for every $n \geq n_{0}$. From inequality (1.3) we have following inclusions

$$
\left\{n: x_{n}<x_{0}-\varepsilon\right\} \subset\left\{1,2, \ldots, n_{0}\right\}
$$

and

$$
\left\{n: x_{n}>x_{0}+\varepsilon\right\} \subset\left\{1,2, \ldots, n_{0}\right\} .
$$

These inclusions implies that $x_{0}-\varepsilon \in L_{s t_{\lambda}}\left(x_{n}\right)$ and $x_{0}+\varepsilon \in U_{s t_{\lambda}}\left(x_{n}\right)$ are satisfied for any arbitrary $\varepsilon>0$.

Hence, $s t_{\lambda}-\inf x_{n}=s t_{\lambda}-\sup x_{n}=x_{0}$. 
Remark 1.7. The converse of Theorem 1.6 is not true, in general. In order to see this let us consider a sequence $\tilde{x}=\left(x_{n}\right)$ as follows:

$$
x_{n}:= \begin{cases}1, & n=\lambda^{2}(k), k \in \mathbb{N} \\ 0, & \text { otherwise }\end{cases}
$$

for any $\lambda=\left(\lambda_{n}\right) \in \Lambda$. It is clear that $s t_{\lambda}-\inf x_{n}=s t_{\lambda}-\sup x_{n}=0$, but the sequence $\tilde{x}=\left(x_{n}\right)$ is not convergent to zero. Let us note that $\tilde{x}=\left(x_{n}\right)$ is $\lambda$-statistical convergent to 0 .

Theorem 1.8. $s t_{\lambda}-\lim x_{n}=x_{0}$ if and only if $s t_{\lambda}-\inf x_{n}=s t_{\lambda}-\sup x_{n}=x_{0}$.

Proof. (Necessity part) Assume that $s t_{\lambda}-\lim x_{n}=x_{0}$. Then for $\varepsilon>0$ the set

$$
\left\{n:\left|x_{n}-x_{0}\right| \geq \varepsilon\right\}
$$

has zero $\lambda$-density. The set in (1.4) can be expressed as a union of two sets as follows

$$
\left\{n: x_{n} \geq x_{0}+\varepsilon\right\} \cup\left\{n: x_{n} \leq x_{0}-\varepsilon\right\}
$$

Hence, from the assumption we have

$$
\delta_{\lambda}\left(\left\{n: x_{n} \geq x_{0}+\varepsilon\right\}\right)=0
$$

and

$$
\delta_{\lambda}\left(\left\{n: x_{n} \leq x_{0}-\varepsilon\right\}\right)=0
$$

for every $\varepsilon>0$. These equations impliy that $x_{0}-\varepsilon \in L_{s t_{\lambda}}(\tilde{x})$ and $x_{0}+\varepsilon \in U_{s t_{\lambda}}(\tilde{x})$ hold for every $\varepsilon>0$. It means that, $s t_{\lambda}-\inf x_{n}=s t_{\lambda}-\sup x_{n}=x_{0}$. is,

(Sufficiency part) Now assume that $s t_{\lambda}-\inf x_{n}=s t_{\lambda}-\sup x_{n}=x_{0}$ holds. That

$$
\sup L_{s t_{\lambda}}(\tilde{x})=\inf U_{s t_{\lambda}}(\tilde{x})=x_{0}
$$

hold. From the usual definition of supremum and infimum there exist $x_{1} \in L_{s t_{\lambda}}(\tilde{x})$ and $x_{2} \in U_{s t_{\lambda}}(\tilde{x})$ such that $x_{0}-\varepsilon<x_{1}$ and $x_{2}<x_{0}+\varepsilon$ hold for any $\varepsilon>0$. From the above inequalities we have following inclusions

$$
\left\{k: x_{0}+\varepsilon \leq x_{k}\right\} \subset\left\{k: x_{2} \leq x_{k}\right\}
$$

and

$$
\left\{k: x_{k} \leq x_{0}-\varepsilon\right\} \subset\left\{k: x_{k} \leq x_{1}\right\} .
$$

Since $x_{2}$ and $x_{1}$ are $\lambda$-statistical upper bound and $\lambda$-statistical lower bound of the sequence, respectively then we have also

$$
\delta_{\lambda}\left(\left\{k: x_{0}+\varepsilon \leq x_{k}\right\}\right)=0,
$$

and

$$
\delta_{\lambda}\left(\left\{k: x_{k} \leq x_{0}-\varepsilon\right\}\right)=0 .
$$

If we combine all facts, then we have

$$
\left|\left\{k:\left|x_{k}-x_{0}\right| \geq \varepsilon\right\}\right|=\left|\left\{k: x_{0}+\varepsilon \leq x_{k}\right\}\right|+\left|\left\{k: x_{k} \leq x_{0}-\varepsilon\right\}\right|
$$

and

$$
\delta_{\lambda}\left(\left\{k:\left|x_{k}-x_{0}\right| \geq \varepsilon\right\}\right)=0 .
$$

Hence, $s t_{\lambda}-\lim x=x_{0}$.

Theorem 1.9. Let $\lambda, \mu \in \Lambda$ such that $\lambda(n) \leq \mu(n)$ holds for all $n \in \mathbb{N}$. If $\limsup _{n \rightarrow \infty} \frac{\mu(n)}{\lambda(n)}<\infty$, then for any $\tilde{x}=\left(x_{n}\right)$

$$
L_{s t_{\mu}}(\tilde{x}) \subset L_{s t_{\lambda}}(\tilde{x}) \text { and } U_{s t_{\mu}}(\tilde{x}) \subset U_{s t_{\lambda}}(\tilde{x})
$$


Proof. Let $l \in L_{s t_{\mu}}(\tilde{x})$ be an arbitrary element. Since $\lambda(n) \leq \mu(n)$ holds for all $n \in \mathbb{N}$, then following inclusion

$$
\left\{k \leq \lambda(n): x_{k}<l\right\} \subset\left\{k \leq \mu(n): x_{k}<l\right\}
$$

and following inequality holds

$$
\left|\left\{k \leq \lambda(n): x_{k}<l\right\}\right| \leq\left|\left\{k \leq \mu(n): x_{k}<l\right\}\right| .
$$

This also implies that

$$
\frac{1}{\lambda(n)}\left|\left\{k \leq \lambda(n): x_{k}<l\right\}\right| \leq \frac{\mu(n)}{\lambda(n)} \frac{1}{\mu(n)}\left|\left\{k \leq \mu(n): x_{k}<l\right\}\right|
$$

Hence, we have $l \in L_{s t_{\lambda}}(\tilde{x})$ under assumption. So, $L_{s t_{\mu}}(\tilde{x}) \subset L_{s t_{\lambda}}(\tilde{x})$ holds. By following same arguments, $U_{s t_{\mu}}(\tilde{x}) \subset U_{s t_{\lambda}}(\tilde{x})$ is obtained easily. So it is left for the reader.

Theorem 1.10. Let $\lambda, \mu \in \Lambda$ be two sequence and $\delta_{\lambda}(\{n: \lambda(n) \neq \mu(n)\})=0$ (or $\left.\delta_{\mu}(\{n: \lambda(n) \neq \mu(n)\})=0\right)$. Then, for any sequence $x=\left(x_{n}\right)$

$$
L_{s t_{\mu}}(x)=L_{s t_{\lambda}}(x) \text { and } U_{s t_{\mu}}(x)=U_{s t_{\lambda}}(x)
$$

hold.

Proof. We will prove only for the set $\delta_{\lambda}(\{n: \lambda(n) \neq \mu(n)\})=0$. The other can be prove similarly way. Let $l \in L_{s t_{\lambda}}(x)$ be an arbitrary element and denote the set $\{n: \lambda(n) \neq \mu(n)\}$ by A. Hence, we have

$$
\begin{gathered}
\left\{k \leq \mu(n): x_{k}<l\right\}=\left(\left\{k \leq \mu(n): x_{k}<l\right\} \cap A\right) \cup\left(\left\{k \leq \mu(n): x_{k}<l\right\} \cap A^{c}\right) \\
\subseteq\left\{k \leq \lambda(n): x_{k}<l\right\} \cup A .
\end{gathered}
$$

where $A^{c}$ denotes the complement of the set $A$. Since $l \in L_{s t_{\lambda}}(x)$ and $\delta_{\lambda}(A)=0$, then we obtain

$$
\delta_{\lambda}\left(\left\{k \leq \mu(n): x_{k}<l\right\}\right)=0 .
$$

That is, $l \in L_{s t_{\mu}}(x)$. By the following since way equality can be prove. So it is ommitted.

If we take $\lambda_{n}=n$ for $n \in \mathbb{N}$, we obtain $U_{s t}(x)$ (the set of statistical upper bounds) and $L_{s t}(x)$ (the set of statistical lower bounds) instead of $U_{s t_{\lambda}}(x)$ and $L_{s t_{\lambda}}(x)$, respectively.

Now we are ready to give foolowing result:

Theorem 1.11. For any $\lambda \in \Lambda, U_{s t_{\lambda}}(x) \subseteq U_{s t}(x)$ and $L_{s t_{\lambda}}(x) \subseteq L_{s t}(x)$.

Proof. Let $l \in U_{s t_{\lambda}}(x)$ be an arbitrary element. Then, the set $\left\{k \leq \lambda_{n}: x_{k}<l\right\}$ has zero $\lambda$-density. The sequence $\lambda=\left(\lambda_{n}\right)$ is an inreasing sequence of natural numbers $\mathbb{N}$ and tends to infinity.

Therefore, we can consider the following sequence

$$
\left(\frac{\left|K_{n}^{\lambda}\right|}{\lambda(n)}\right)_{n \in \mathbb{N}}
$$

as a subsequence of

for any $K \subset \mathbb{N}$.

$$
\left(\frac{\left|K_{n}\right|}{n}\right)_{n \in \mathbb{N}}
$$


Hence, the set $\left\{k \leq n: x_{k}<l\right\}$ has zero asymptotic density. So, $l \in U_{s t_{\lambda}}$. The second inclusion can be proved by the following same steps. It is left for the readers.

Corollary 1.12. For any $\lambda \in \Lambda$ and sequence $x=\left(x_{n}\right)$ we have

$$
s t-\inf x_{n} \leq s t_{\lambda}-\inf x_{n} \leq s t_{\lambda}-\sup x_{n} \leq s t-\sup x_{n} .
$$

Corollary 1.13. For any $\lambda=\left(\lambda_{n}\right) \in \Lambda$ we have that

$$
\text { st }-\lim x_{n}=l
$$

implies

$$
s t_{\lambda}-\lim x_{n}=l .
$$

Inverse of the corollary is not true.

\section{Conclusion and Recommendation}

In this paper, Theorem 1.6 and Theorem 1.8 are given as an application of $s t_{\lambda}-\sup$ and $s t_{\lambda}-$ inf. It is seen that equality of $s t_{\lambda}-\sup$ and $s t_{\lambda}-$ inf necessary but not sufficient for existence of classical limit but necessary and sufficient for existence of $\lambda$-statistical limit.

As a continuation of this work one can define $\lambda$-statistical boundedness and interested in with the space of $\lambda$-statistical bounded sequences space:

$l_{\infty}^{s t_{\lambda}}=\left\{\tilde{x}:\right.$ there exists $M>0$ such that $\left.\delta_{\lambda}\left(\left\{n \in \mathbb{N}:\left|x_{n}\right|>M\right\}\right)=0\right\}$.

\section{Acknowledgment}

We would like to thank referees for their valuable contributions of the paper.

\section{REFERENCES}

[1] Altınok. M, Küçükaslan. M, A-Statistical Supremum-Infimum and A-Statistical Convergence, Azerbaijan Journal of Mathematics, Vol.4, No.2, pp.43-57, (2014).

[2] Altınok. M, Küçükaslan. M, Ideal Limit Superior-Inferior, Gazi University Journal of Science, Vol.30, No.1, pp.401-411, (2017).

[3] Altınok. M, Porosity Supremum-Infimum and Porosity Convergence, Konuralp Journal of Mathematics, Vol.6, No.1, pp.163-170, (2018).

[4] Fast. H, Sur la convergence statistique., Colloq. Math, Vol.2, pp.241-244, (1951).

[5] Fridy. J. A, On statistical convergence, Analysis, Vol.5, pp.301-313, (1985).

[6] Küçükaslan. M, Altınok. M, Statistical supremum infimum and statistical convergence, Aligarh Bulletin of Mathematics, Vol.32, pp.1-16, (2013).

[7] Milan. P, Density and related topics, Mathematics Institute Slovak Academic of Sciences (2017).

[8] Schoenberg. I. J, The integrability of certain functions and related summability methods, matrix characterization of statistical convergence, Amer.Math., Vol.66, pp.361-375, (1959).

[9] Steinhaus. H, Sur la convergence ordinaire at la convergence asymptotique, Colloq. Math., Vol.2, No.1, pp.73-74, (1951).

[10] Zygmund. A, Trigonometric series, 2nd., Ed. Vol. II, Cambridge Univ. press, London and New York (1979).

(M. Altinok) Tarsus University, Faculty of Engineering, Department of Natural and Mathematical Sciences, Mersin, Turkey

(U. Kaya) Mersin University, Faculty of Science and Art, Department of Mathematics, Mersin, Turkey 
(M. Küçükaslan) Mersin University, Faculty of Science and Art, Department of Mathematics, Mersin, Turkey

Email address, M. Altınok, U. Kaya, M. Küçükaslan: mayaaltinok@tarsus.edu.tr, umutcanmath@gmail.com, mkkaslan@gmail.com 